\title{
Optimization Design Of Setback Generator For Initiating
}

\section{Explosive Devices}

\author{
Qiao $\mathrm{Lu}^{\mathrm{a}}$, Liming $\mathrm{Li}^{\mathrm{b}}$ and Guofu $\mathrm{Yin}^{\mathrm{c}}$ \\ State Key Laboratory of Applied physic-Chemistry Research, China \\ a1185256995@163.com, bbiq213@163.com, cyinguofu521@126.com
}

\begin{abstract}
This paper describes an optimized design of setback generator based on the smart miniature initiator's demand for physical power, Through the Hopkinson bar impact experiments, we conducted a comparative study of multiple sets of high overload experiments and concluded that axial magnetized permanent magnets with the homopolar opposite and distribution winding can improve generator's power generation efficiency to certain degree. When axial magnetized permanent magnets with homopolar opposite and distribution winding are used to assemble the optimized setback generator, it can detonate ignition head consistently at $20000 \mathrm{G}$ acceleration.
\end{abstract}

Keywords: Initiating explosive devices; physical power source; setback generator.

\section{Introduction}

The complexity of the modern battlefield requires higher performance of weapon system, as an important component of the weapon system,the initiating explosive devices being developed toward to the intelligent, miniaturization direction[1]. The missile-borne power supply release electric energy as the acceptable energy for stimulating Initiating Explosive, currently most of the Initiating Explosive uses missile-borne battery as their Power supply. With the continuous development of Initiating Explosive, also spawned a variety of ways to provide power in addition to onboard battery power supply[2].

Physical power is being paid more attention because it has a lot of outstanding features such as non-destructive testing, reusability, long-term storage, withstand high and low temperatures, high security and others[3]. As an important physical power,setback generator plays an important role in Initiating Explosive applications. The setback generator is a pulsed power supply.When the device is under the influence of the recoil,the magnet in the device is doing axial linear motion relative to the coil. So that the magnetic flux through the coil is changed, which generate induced electromotive force and induced current in the coil. But due to the setback generation device using pulse power, which produces less energy. A cylindrical structure setback generator has been designed by Korea Agency For Defense Development, which have a diameter of $17.7 \mathrm{~mm}$, a height of $4.1 \mathrm{~mm}$, and a weight of $6.0 \mathrm{~g}$. This setback generators test results show that a voltage of $4.6 \mathrm{~V}$ was charged on a capacitor of $100 \mathrm{~F}$ within the charging time of $0.56 \mathrm{~ms}$ under $30000 \mathrm{~g}$ accelerations [3].

\section{Setback Generator Ignition System Design and Theoretical Basis}

This paper describes a diagram of missile-borne setback generator which provides power design. In the system, the setback generator firstly generates pulse voltage in the function of recoil, and then pulse voltage is stored in the 
capacitor through the rectifier device and a voltage stabilizing device. Capacitor power can supply power for the control circuit. And explosive initiation energy can also be provided by it in the case of an open security. The whole system is controlled by the microcontroller.

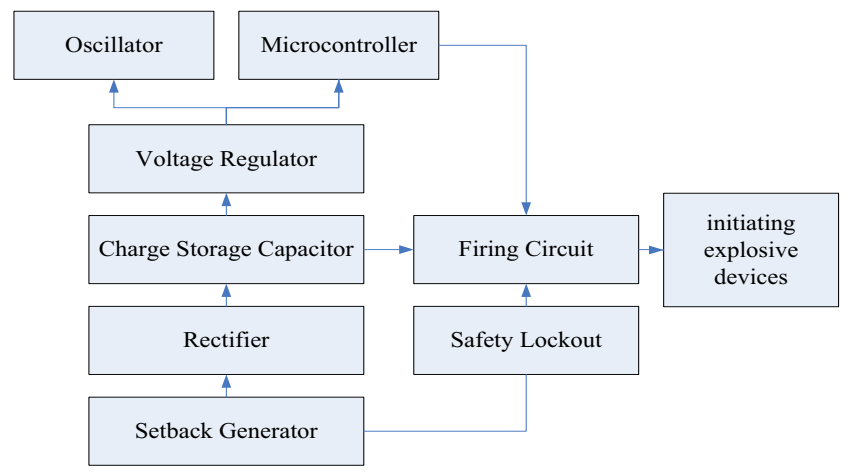

Fig. 1. Block diagram of power supply system

The setback generator is the key of the whole power supply system described above. When setback generator in working process, because the permanent magnet around the magnetic field distribution is non-uniform, and there is a relative motion between the coil and the magnet,through analysis of the Faraday's law of electromagnetic induction we can conclude that in this producing energy process, the electromotive force generate both induced electromotive force and motional electromotive force.In this paper, any one turn coil' $\mathrm{s}$ induce electromotive force generated by the expression:

$$
E_{l}=E_{i}+E_{m}=-\int_{A} \frac{\partial \vec{B}}{\partial t} \cdot d \vec{s}+\oint_{L}(\vec{v} \times \vec{B}) \cdot d \vec{L}
$$

For the $\mathrm{N}$ turns of the coil, the total induced electromotive force is generated in this process equals to the sum of each single coil induced electromotive force, expressed as:

$$
E=\sum_{i=1}^{N} E_{i}
$$

\section{Experiment and Analyses}

The disadvantage of low energy output has been restricting the development of setback generator, the experiments will be conducted to design new structure for the permanent magnet part and the coil part of the device, which aims to improve its performance. The reliability of initiating explosive device will be improved when it is integrated with optimized setback generator [5].

Hopkinson Pressure Bar (SHPB) is one of the most basic experimental apparatus in the material dynamics.It mainly measures the dynamic stress and strain relationship of the material,and it is widely used in the dynamic mechanical properties of the materials at home and abroad[4].

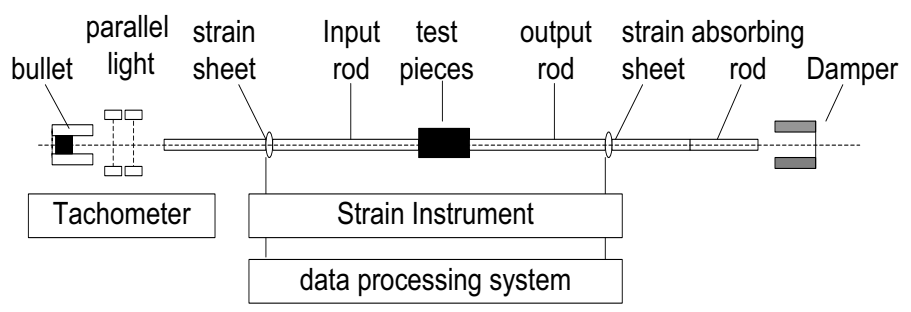

Fig. 2. Hopkinson bar system diagram 
In this study we need to use the input rod of Hopkinson bar to directly hit impact on the setback generator in order to roughly simulate the ammunition launching process. When setback generator in the high acceleration of the environment, the permanent magnet and the coil in it will produce a high speed relative displacement and produce energy. The energy waveform is recorded and is used for comparison and analysis.

\subsection{Comparative experiment of permanent magnet structure}

Magnetization direction of the permanent magnets is generally divided into two types, they are radial and axial magnetization. This attribute has already been decided in the production process of permanent magnet. In addition to the magnetization direction, the topology of the permanent magnet is also an important factor affecting the output of setback generator. Based on their different topologies axially magnetized ,permanent magnets can be further divided into: a single length of the permanent magnet, two short permanent magnets of different poles opposite and two short permanent magnets with the homopolar opposite.

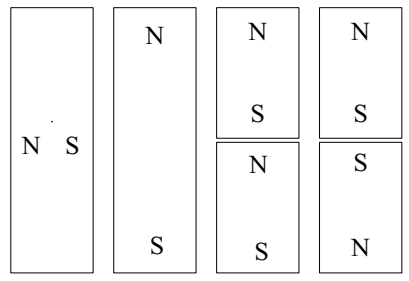

Fig. 3. Permanent magnet for testing

According to the different magnetizing direction and topology, we designed above four kinds of permanent magnet structures for comparison. We conducted the Hopkinson bar impact test without changing the other parameters. The output cable of the setback generator was connected with the Oscilloscope in order to collect no load voltage. And the data was compared and analyzed.

Experimental results and analysis: Based on the magnetization direction of the permanent magnets and the topology will be four groups of experiments were conducted. The voltage waveforms of the measured waveforms are shown in the following figure.

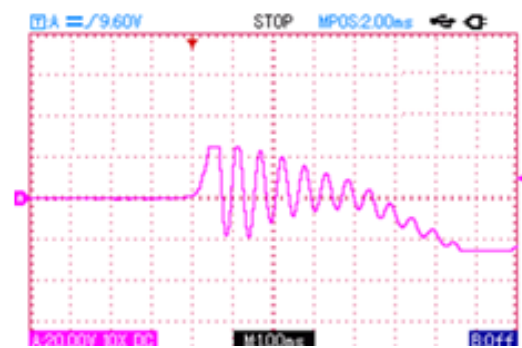

Fig. 4. Radial magnetization

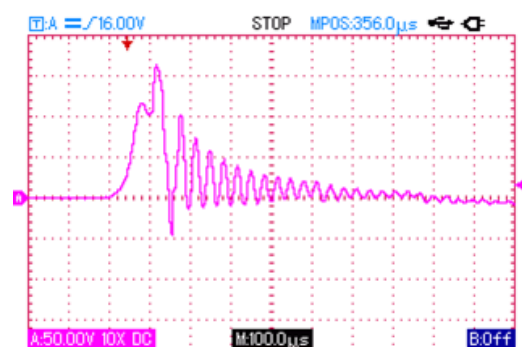

Fig. 6 Different polar opposite

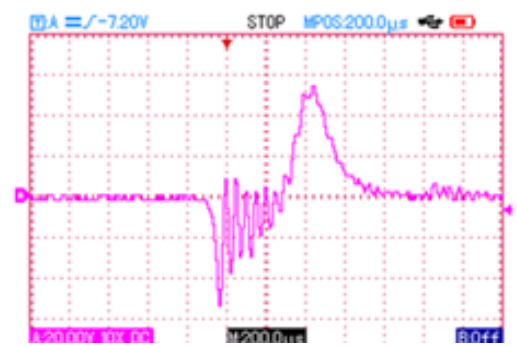

Fig. 5. Axial magnetization

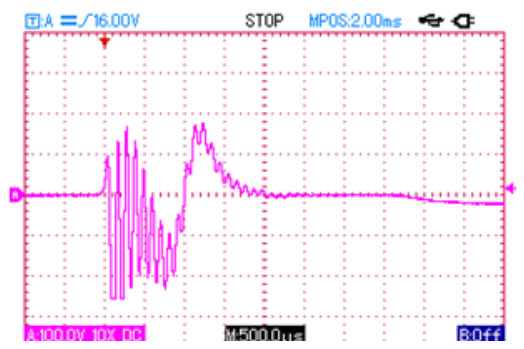

Fig. 7 Homo polar opposite 
We made some conclusions through experiment:

(1)The setback generators which use the radial magnetized permanent magnet produce less energy, but longer discharge duration.

(2) Using axial magnetized permanent magnet setback generation compared to using a radially magnetized permanent magnet setback generation will produce more energy, but relatively short duration of the discharge.

(3) For axially magnetized permanent magnet, the setback generator which use magnets with the homopolar opposite have shorter discharge time, but the discharge waveform more tidy, complete and higher quality discharge.

\section{2 comparative experiments of coil winding structure}

Depending on the circumstances, coil winding method can be divided into the distribution winding method and non-distribution winding method. Multi slot and multi group coil mode is used in the distribution winding method, using series connection between each group. It found that using the distribution winding method can to some extent improve the generation efficiency of setback generator in the early simulation work, this conclusion is also required Hopkinson Bar experiments to validate it.

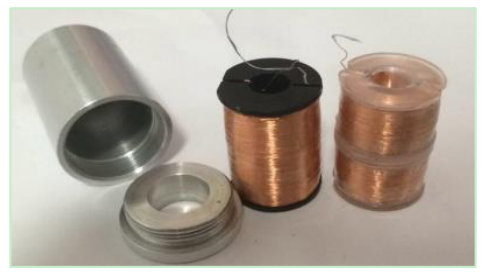

Fig. 8. Distribution winding and non-distribution winding

As shown in the above two kinds of different winding ways of the coil. In order to reduce the eddy current effect in power generation, the plastic is used as the skeleton of the coil. Non distribution winding height $16 \mathrm{~mm}$, diameter $10 \mathrm{~mm}$, with a diameter of $0.06 \mathrm{~mm}$ enameled copper wire winding, winding turns 8000 turns, the resistance is $2.2 \mathrm{~K} \Omega$.The distribution winding on the right using two smaller superposition of coil windings in series, each winding coil height $8 \mathrm{~mm}$, diameter $10 \mathrm{~mm}$, with a diameter of $0.06 \mathrm{~mm}$ enamelled copper wire, is wound turns 4000 turns, the resistance is $1.1 \mathrm{~K} \Omega$.

In order to compare two winding method, the axial charge magnetism homopolar opposite permanent magnets was selected as the experiment permanent magnet. Respectively, we use distributed windings bad and non-distributed coil winding to conduct Hopkinson bar impact test. The results were compared and analyzed.

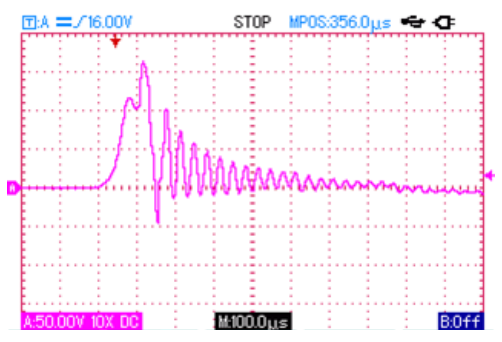

Fig. 9. Non distribution winding

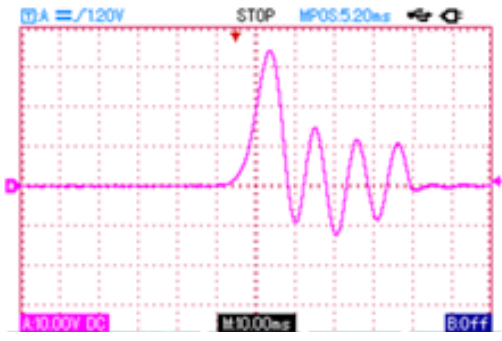

Fig. 10. Distribution winding

We made some conclusions through experiment:

(1) The setback generator with distributed windings will obviously produce a higher voltage, the energy conversion efficiency is higher.

(2) The discharge time of the setback generator using the distributed winding is longer than that of the setback generation using the non-distributed winding. 
(3) The voltage waveform of the setback generator with the distribution winding is more complete. It shows that the distribution winding method can effectively eliminate the harmonic and improve the power quality.

\section{3 setback generation device detonated igniter test}

According to the permanent magnet experiment and coil experiment, we selected axial charge magnetism homopolar opposite permanent magnet and a distribution winding coil to integrate an optimized setback generator, and then connect the setback generator with follow-up circuit and ignition igniter test.

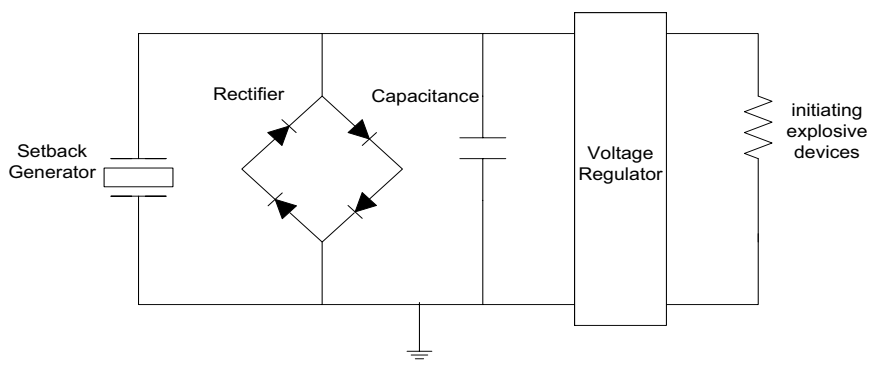

Fig.11. Ignition Experiment circuit diagram

Hopkinson bar produced an acceleration $20000 \mathrm{~g}$ overload impact generating device. The energy generated flow into the capacitor through the bridge rectifier, and then detonated igniter after the regulator. A total of five trials were carried out, and all the ignition was successfully initiated.

\section{Summary}

Changing the structure of permanent magnet and the coil winding method can effectively improve production efficiency of the setback generating device. Most favorable factor for power generating efficiency is to have permanent magnet with axial magnetizing direction and homopolor opposite topological structure. As far as the choice of coil winding method is concerned, the most favorable factor is to have the distribution winding way. When axial magnetized permanent magnets with homopolor opposite and distribution winding are used and integrated to assemble the generator, it can detonate ignition head consistently under $20000 \mathrm{G}$ acceleration.

\section{References}

1. Amirtharajah, Chandrakasan, Sci. Forum, 22-23 (2011)

2. Sang-HeeYoon, submitted to Journal of Materials Research (2005)

3. Cheng Hok, Aw, Yong Lim Thoma, U.S. Patent 656, 471 (2003)

4. Hu Shi-sheng, Wang Dao-rong, Journal of Engineering Mechanics, (2001)

5. Zhao Ge-xiao, Chen Zhi-yuan, Journal of Engineering Mechanics, (2009) 